\title{
Implementation of Newborn Hearing Screening in Rizal, Philippines, and Northern California: Lessons Learned
}

\author{
Carlos Diego A. Rozul, MClinAud, ${ }^{1}$ Ernesto R. Gregorio, Jr., MSc ${ }^{2}$ and Charlotte M. Chiong, MD, $\mathrm{PhD}^{3}$ \\ ${ }^{1}$ Research Implementation and Development Office, College of Medicine, University of the Philippines Manila, Philippines; \\ ${ }^{2}$ Department of Health Promotion and Education, College of Public Health, University of the Philippines Manila, Philippines; \\ ${ }^{3}$ Department of Otorhinolaryngology, College of Medicine, University of the Philippines Manila, Philippines
}

\begin{abstract}
Objective. The universal newborn hearing screening program has been implemented in the Philippines for the past ten years. However, screening rates in the country are still low. The current study aimed to describe the universal newborn hearing screening program (UNHSP) delivery system in Rizal, Philippines, and Northern California.
\end{abstract}

Methods. The study utilized a case study research design using data triangulation of FGD, KII, and document review to characterize and compare the implementation of the Universal Newborn Hearing Screening Program in Rizal Province and Northern California.

Results. Several differences were found in the protocols for newborn hearing screening in Rizal, Philippines, and Northern California, including centralization of the program, availability of surveillance data, screening protocols, and tracking system.

Conclusion. There is an immense need to disseminate universal newborn hearing screening among healthcare practitioners and create a system to monitor and evaluate real-time data.

Keywords: universal newborn hearing screening program, Hearing for Life Project, health service delivery

\section{INTRODUCTION}

Corresponding author: Carlos Diego A. Rozul, MClinAud Research Implementation and Development Office College of Medicine

University of the Philippines Manila, Philippines

Taft Avenue, Ermita, Manila 1000, Philippines

Email: carozul@up.edu.ph
In 2009, the Philippines enacted the Universal Newborn Hearing Screening and Intervention Act. ${ }^{1}$ This feat made the Philippines one of the few countries with legislation for a universal newborn hearing screening program. ${ }^{2}$ Evidence shows that a universal newborn hearing screening program (UNHSP) increases early diagnosis and habilitation of hearing loss which can prevent speech and language delays, reduce the age of cochlear implantation, is cost-effective with a tracking system. ${ }^{3-9}$ Similarly, Santos-Cortez and Chiong found that universal newborn hearing screening was costeffective in the Filipino setting despite initial expenses. ${ }^{10}$

Leading in implementing the UNHSP, different hospitals reported having similar findings regarding their experiences with newborn hearing screening, such as detecting hearing loss in well-babies, higher prevalence of hearing loss among high-risk infants, and difficulties in following up experiences of other developing countries. ${ }^{6,11-13}$ Novel methods of newborn hearing screening were also developed in response to the legislation of the universal newborn 
hearing screening program to accommodate regions without a newborn hearing screening device. ${ }^{14,15}$ Despite this, the 2018 newborn hearing screening registry shows only 33,127 infants (1.96\%) screened out of 1,668,120 live births. ${ }^{16}$

Fang et al. explored factors in successfully implementing the universal newborn hearing screening program, including a mandatory newborn hearing screening policy, low to no intervention cost, system design, decision making, funding, and a concrete implementation plan. ${ }^{17}$ Routine linkage with health insurance and tracking systems were also found to improve follow-up for detection of bilateral hearing loss. ${ }^{9} 18$ Awareness and attitudes of the public and the knowledge and attitudes of the healthcare practitioners also play a role in the program's effectiveness, especially in rural and geographically disadvantaged areas.,6,19 Maternal attitudes toward universal newborn hearing screening were affected by their knowledge of the hearing screening process, risk factors, availability of resources, cost, and extended family knowledge and attitudes. ${ }^{3,6,20-24}$

Telehealth is one of the more recent innovative strategies to improve the coverage of newborn hearing rescreening. It has been used to bridge the human resource gap in health service delivery. In Northern California, the University of California Davis Health System (UCDHS) has played an integral role in delivering healthcare services to rural and urban areas. The institution serves 33 counties across 17,000 square kilometers being also the only level 1 adult and pediatric trauma center. The region has benefited from the telemedicine program that UCDHS provides since the mid-1990s. ${ }^{25}$ Since then, the program has positively impacted reducing patient travel time, patient travel cost, and environmental pollutants; it has proven costeffective to implement telemedicine consultation than telephone consultations. ${ }^{26,27}$ Swanepoel and Hall reported a recent focus on tele-audiology services and research in remote sites, diagnosis, and intervention. ${ }^{28}$ Through the Philippine-California Advanced Research Institutes, academic institutions are collaborating towards increasing the rates of newborn hearing screening in the country, especially in geographically disadvantaged areas, through novel technology and telehealth.

In 2010, Northern California experienced a 22\% loss to follow-up after getting refer results from newborn hearing screening services. However, after the implementation of the tele-audiology diagnostic evaluations, loss to followup was reduced to $0 \% .{ }^{29}$ The current study aims to describe lessons learned from Northern California which can be applied to improve the implementation of the UNHSP in the Philippines.

\section{MATERIALS AND METHODS}

\section{Study Design}

The present study utilized a case study design to describe the implementation of the UNHSP in Northern California and the Rizal province. This study aims to extract lessons learned from implementing the UNHSP using Focus Group Discussions (FGDs), key informant interviews (KIIs) of UNHSP coordinators, review of protocols and regulations of UNHSP in Northern California and the Philippines.

\section{Study Sites}

Northern California was chosen as a model in implementation due to its utilization of telehealth in improving its performance of the Early Hearing Detection and Intervention Act. This may serve as an ideal model for the Hearing for Life Project, where the University of California, Davis, and Berkeley are collaborating agencies. Rizal province was chosen as a comparison due to the limited number of certified universal newborn hearing screening centers in the area, most of which are located within the periphery of Metro Manila.

\section{Participants}

The current study investigated the implementation of UNHSP in the Philippines and Northern California. In the context of this study, purposive sampling was used to recruit study participants. Healthcare workers involved in the delivery of newborn hearing screening were primarily targeted to participate. There were five midwives from birthing homes, twelve rural healthcare workers from rural health units, three Pediatric consultants, five nurses from primary and secondary hospitals, and three OBGYN consultants from tertiary hospitals in Rizal Province included in the study.

Coordinators from the Northern California Region Hearing Coordination Center (HCC) and nurses who oversee the newborn hearing screening program in the University of California Davis Medical Center (UCDMC) and other hospitals were also invited to share experiences regarding newborn hearing screening delivery using snowball sampling. There were six KIIs conducted.

\section{Data Collection}

An authorized letter endorsed by the Region IV-A Office of the Department of Health (DOH IV-A) was sent to 43 health institutions in Rizal province that invited key participants to take part in the study. However, only 39 institutions were located at the designated addresses provided by the DOH IV-A registry. A total of 60 healthcare practitioners were invited to participate in homogenous FGDs grouped by profession. The FGDs were conducted from February to March 2018.

In California, subject matter experts were invited to participate in a key informant interview (KII) through email. Managers from the HCC were invited but refused to participate. When approved, coordination with the participants was done to schedule a face-to-face interview at the participant's preferred venue. The discussion revolved around their experiences in implementing the program. The KIIs were conducted from September to November 2018. 
Each participant was given an informed consent form that explains the study's rationale, potential risks and benefits of participating, access to results, and the confidential and anonymous treatment of collected data. The primary researcher developed the topic guide for data collection and was reviewed and approved by the senior author. The guide questions (Appendix A) for the Rizal data collection were composed of questions regarding the effects and implications of hearing loss in early stages of life, eligibility for newborn hearing screening, healthcare practitioners' perceptions regarding their role in the implementation of the Newborn Hearing Screening Program, and the challenges faced and perceived solutions toward implementing the program in their respective institutions. The guide questions (Appendix B) for California participants aimed to contextualize how UNHSP was implemented and explore previous gaps and solutions.

A document review was also conducted to collate documentation on implementing the universal newborn hearing screening program in the study sites. Legislation for each study site was retrieved from https://www.officialgazette. gov.ph/ for Rizal, Philippines, and https://leginfo.legislature. ca.gov/ for Northern California. Public websites for the newborn hearing screening programs were also included in the review to extract information on the screening methods, screening rates, and innovations in the newborn hearing screening program. Manuals of operation were also included in the review.

\section{Data Analysis}

A document review was also conducted to compare the implementation of the UNHSP between the two sites according to regulation, history, government support, methods, and innovations.

Multimedia recordings from the FGDs and KIIs were transcribed. The principal investigator did a thematic analysis to synthesize the findings from the collected data with the help of the qualitative data analysis program NVIVO 12. Data were encoded as practices, challenges, and solutions. Themes were then identified by reviewing the relationships of the answers of each FGD and KII. Initial coding was discussed with co-investigators to reach an agreement.

These themes were triangulated with the available surveillance data, legislation, and the provided program guidelines in the UCDMC and the Manual of Operations of the Universal Newborn Hearing Screening in the Philippines.

\section{RESULTS}

Sixty healthcare practitioners were invited to participate from Rizal province. However, only 28 were able to join the study, where seven were directly involved in the newborn hearing screening process, and two were managing the service in their institution. None were certified by the
NHSRC. The participants included rural health workers, private hospital nurses, government hospital OBGYN and Pediatric consultants, and private birthing home midwives. The FGDs were held in six separate sessions.

The HCC did not participate due to state restrictions in sharing data. Out of the twelve intended participants from the UC Davis Medical Center, only four agreed to participate in the KIIs. Two participants were from other hospitals in Northern California. Participants included two hearing screening coordinators, a pediatric audiologist, a consulting audiologist, and a newborn hearing screening outsourcing company manager. Two participants were directly involved in the newborn hearing screening process, while three had experience managing the program.

The legislation was found in both settings supporting universal coverage of newborn hearing screening and intervention. Websites of the universal newborn hearing screening program of each site showed the program's history. The program in Rizal is regulated centrally as part of the national program through the Newborn Hearing Screening Reference Center (NHSRC). Meanwhile, in California, services related to newborn hearing screening and intervention are managed by three entities, namely the Hearing Coordination Center (HCC), California Children's Services (CCC), and the Early Start Program (ESP). It was also seen that NHSRC allows OAE and AABR devices in newborn hearing screening, but not in conjunction. California, however, allows various screening protocols across institutions using $\mathrm{OAE}$ and $\mathrm{AABR}$ in different circumstances and combinations. Table 1 summarizes the characteristics of the implementation of the UNHSP in Rizal province and Northern California from the review of records.

\section{Implementation in the Rizal Province}

The UNHSP in Rizal province is regulated by a central body, the Newborn Hearing Screening Reference Center (NHSRC). It assists government and non-government agencies in implementing, training, and awareness campaigns related to the program. This includes defining and recommending protocols and methods in newborn hearing screening, managing the UNHSP registry, and certification personnel and centers for screening, diagnostic testing, and intervention. Despite this, the Department of Health has yet to require UNHSP to be part of hospital certification. However, upon request, the NHSRC was not able to provide the screening rate of Rizal province.

The NHSRC has recommended using otoacoustic emission (OAE) and automated auditory brainstem response (AABR) devices to be used in UNHSP together with a stop criteria. Given this, most participants who provided UNHS were not aware of these protocols.

Legislation of the UNHSP in Rizal is present through the Universal Newborn Hearing Screening and Intervention Act of 2009 (RA9709), ensuring that all newborns have access to early hearing loss screening, diagnosis, and 
Table 1. Comparison of UNHSP Implementation in Rizal and Northern California

\begin{tabular}{|c|c|c|}
\hline & Rizal & Northern California \\
\hline Regulation of Program & $\begin{array}{l}\text { Centralized } \\
\text { - Newborn Hearing Screening Reference Center } \\
\circ \text { Recommendations for protocols and procedures } \\
\circ \text { Registry Database Management } \\
\circ \text { Certification of Centers and Personnel }\end{array}$ & $\begin{array}{l}\text { Decentralized } \\
\text { - Hearing Coordination Center } \\
\circ \text { Patient Tracking } \\
\circ \text { Certificate of Program / Quality Assurance } \\
\circ \text { Data Management } \\
\text { - California Children's Services } \\
\circ \text { Certification of Assessment and Intervention Centers } \\
\text { - Early Start Program } \\
\circ \text { Provision of Intervention Services }\end{array}$ \\
\hline $\begin{array}{l}\text { Year Started Task Force for } \\
\text { Newborn Hearing Screening }\end{array}$ & 2007 & 1993 \\
\hline Screening Rate & No available record & $97 \%(2013)$ \\
\hline Government Support & $\begin{array}{l}\text { Legislation } \\
\text { - Universal Newborn Hearing Screening and } \\
\text { Intervention Act of } 2009 \\
\circ \text { Mandatory that all newborns shall be screened } \\
\text { for hearing }\end{array}$ & $\begin{array}{l}\text { Legislation and Funding } \\
\text { - Newborn and Infant Hearing Screening, Tracking, and } \\
\text { Intervention Program (1998, revised in 2006) } \\
\circ \text { Revision made NHSP mandatory to all hospitals (not } \\
\text { only CCS-certified) }\end{array}$ \\
\hline Method & Predominantly using OAE devices, but allows AABR & Utilizes both $A A B R$ and $O A E$ \\
\hline Innovations Unique to Area & None specific for Rizal & $\begin{array}{l}\text { HCC } \\
\text { - Intended to eliminate loss to follow-up between } \\
\text { screening, diagnosis, and intervention }\end{array}$ \\
\hline
\end{tabular}

intervention. However, majority of the participants in the FGDs claimed to not know about the law. However, those who claimed to know about the law and did not have UNHS in their institution did not refer to institutions that offered the service.

\section{Implementation in Northern California}

In contrast to Rizal province, the provision of UNHSP and its subsequent services are decentralized in Northern California. The Department of Health Care Services (the equivalent of the Department of Health in the Philippines) provides quality indicators, adaptable informational materials, and forms for the implementation of UNHSP. The HCC is a California-only institution created to track patients, ensure compliance to quality indicators, manage registry databases to eliminate loss to follow-up between screening, diagnosis, and intervention. Individual institutions provide personnel training and development of protocols and procedures for the UNHSP, where the HCC evaluates the institution's performance quarterly, which impacts insurance claims. The California Children's Services regulates the assessment and intervention centers to provide nearby referrals for patients. At the same time, the Early Start Program oversees intervention services such as speech therapy.

The Newborn and Infant Hearing Screening and Intervention Act were first enacted in 1998, where UNHSP was first made mandatory to CCS-certified hospitals. Favorable program outcomes prompted the revision of the law in 2006, where the UNHSP became compulsory for all hospitals and the HCC was established.

\section{Challenges and Solutions in Rizal}

"So sabi ng isang ano na-attendan ko na Pedia, dapat daw since mahal naman daw talaga yang Hearing Test na yan, turuan nalang daw mga midwife na pumalakpak." (A pediatrician in a seminar I attended told us that since newborn hearing screening is expensive, midwives should be taught to clap instead) - 49, female, Midwife, and Birthing Home Owner

Building upon the findings by Rozul, Gregorio, and Chiong's $\mathrm{s}^{30}$, one of the prevalent challenges in Rizal include the lack of specific knowledge on implementing UNHSP; this includes knowledge on risk factors, age of hearing detection, stop criteria, submission of records to NHSRC, and referrals after the detection of hearing loss. Participants of the FGD suggested the provision of directories of diagnostic and intervention centers and fact sheets. Some were also receptive to the idea of e-learning modules for UNHSP procedures and protocols.

"We cannot implement anything na wala [( We cannot implement anything that there is none) referring to the absence of in-house UNHSP.])."

- 42, female, Pediatrician Consultant

The participants also expressed a lack of ownership in managing the UNHSP in their institution, suggesting a lack of clear roles and expectations in informing parents of the program and implementing it in their respective institutions. Through an information dissemination protocol, the roles of each professional can be clarified in the implementation of the UNHSP. 
"Nandito na kami sa ganitong trabaho, although

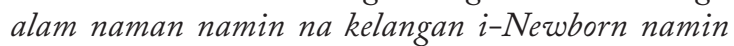
ang bata as soon as possible. Wala lang talaga kaming contact." (We're in this line of work, although we know that the child should be screened as soon as possible. We really don't have contacts) - 36, female, Midwife and Clinic Owner

Lastly, the lack of accessibility to training and services was one of the most influential factors in implementing the UNHSP in Rizal province, cited the participants. Less than half of the participants admitted that there was a certification needed as an institution and to work as the personnel of the UNHSP. More than half expressed that they did not know where the nearest hearing screening, diagnostic, and intervention centers were. Less than half who resorted to outsourcing their UNHS shared their difficulty coordinating with parents to get screened as the third-party provider is only available once a week. The HeLe project is also an opportunity to solve this challenge through a lower-cost teleaudiology-capable AABR device. Table 2 summarizes the challenges and solutions in the delivery of the UNHSP.

\section{Challenges and Solutions in Northern California}

Some hospitals provide newborn hearing screening services through their staff. This includes a detailed protocol and well-defined roles for those involved in managing infants identified with hearing loss. However, some participants expressed their inconvenience with double charting through the hospital's electronic medical records and the state's data management system for newborns. The NICU hearing screening coordinator shared her experience with the HCC's efforts to integrate electronic charting systems and the state's data management system and how it was scrapped.

"To be honest, it's probably a financial decision by the hospital because we're not RNs [Registered Nurses]. We're certified hearing screeners, so we're not taking away some other responsibilities of an $R N$ as she's not spending time doing a hearing screen. So a lot of the time, the hospital comes to us for financial reasons. "- 50, female, Hearing Screening Outsourcing Manager

Outsourcing the service has gained increasing popularity. To reduce cost, personnel responsibilities and still provide the mandated newborn hearing screening services, some hospitals establish a contract with outsourcing companies responsible for implementing and monitoring the program. When an institution changes providers for newborn hearing screening, the HCC gives a 90-day grace period to re-establish the program before it is certified. A hearing screening outsourcing manager of eight private hospitals in Northern California shared that it was common for hospitals to be initially skeptical of their services but eventually buy into their "no hospital cost" model. They only charge the amount that insurance companies would reimburse for the service, unlike their competitors. They also have an established relationship with a hearing screening device manufacturer, which supplies all their hospital sites and gives quick service when a device needs maintenance. This specific company assures competency of their staff through online training modules provided by the National Center for Hearing Assessment and Management, and attend the staff orientation on newborn hearing screening for each hospital site their staff will do the procedure. Each is also closely monitored by the regional manager for their performance.

Tele-audiology was used to reduce the previous $22 \%$ loss to follow up rate to $0 \%,{ }^{31}$ but was not sustained after the grant period was finished due to a lack of insurance reimbursement system for tele-audiology practice. Furthermore, the program became more time-consuming for the audiologists due to the limited skills of the technician from offsite.

There was difficulty following up with parents of NICU babies referred to since they reportedly get admitted to the hospital often and miss their appointment. Moreover, coordination with transferees from different hospitals was complex due to incomplete transfer forms and no direct contact with the primary care provider, making follow-up with the patient less likely. Families who move residence when an appointment is due were also cited as a challenge they pass onto the HCC.

\section{DISCUSSION}

There are several differences in the implementation, challenges, and solutions of the universal newborn hearing screening services in Rizal and California. First, its regulation, compared to California, surveillance and quality assurance of newborn hearing screening services are centralized via the NHSRC. This in contrast to the decentralized law of

Table 2. Comparison of UNHSP Challenges and Solutions in Rizal and Northern California

\section{Rizal}

Challenges and Solutions
Lack of Specific Knowledge on Implementing UNHSP

- E-learning modules, fact sheets, directories

Lack of Information Dissemination Protocol

- Information/education campaign and materials

Lack of Access to Services ${ }^{30}$

- Outsourcing (not staffed every day), HeLe Project Device

\section{Northern California}

Loss to Follow-up After Screening

- Tele-audiology, successful, but not integrated due to lack of billing policy

Double Charting

- Integration of state data management system and electronic charting system, unsuccessful due to glitches

Transfers from Outside the Institution System

- Contact the HCC, refine patient transfer requirements 
its and related services in California. This is also reflective of the method and screening protocols used in each setting. The NHSRC and HCC utilize both OAE and AABR screening methods. However, the NHSRC allows only a set screening protocol using either $\mathrm{OAE}$ or $\mathrm{AABR}$ per screening site compared to California, wherein $\mathrm{OAE}$ and $\mathrm{AABR}$ screening protocols vary among screening sites.

Second, both settings have government support for the universal newborn hearing screening program through legislation. However, California's legislation is more comprehensive, including tracking patients referred for diagnostic and intervention services. This may be attributed to the state's vast experience in implementing the newborn hearing screening program, starting a task force as early as 1993 compared to 2007 in the Philippines. Moreover, the availability of surveillance data in the region differed. The Department of Health Care Services in California was able to provide surveillance data as latest as 2013. However, the NHSRC was not able to produce surveillance data for Rizal. This reflects findings by Mackey and colleagues ${ }^{31}$, which shows that quality measures mainly were unavailable for universal newborn hearing screening programs across 47 selected countries.

Lastly, there is a stark difference in the challenges encountered between the two settings. Mainly, there is a lack of awareness, access, and ownership in delivering newborn hearing screening services in Rizal. As compared to California, wherein the challenges encountered were procedural and went beyond the delivery of initial screening services. This is reflective of the $97 \%$ screening rate in California.

\section{CONCLUSION}

The current study aimed to characterize the implementation of UNHSP in Rizal province and Northern California. As with previous findings $s^{30}$, there is a significant need to disseminate information about newborn hearing screening among healthcare practitioners, including clear roles in implementing the program and directories for confirmatory diagnosis and intervention. This is in conjunction with the lack of regional surveillance data that can be the basis for innovations and interventions for service delivery.

KIIs from Northern California gave insight into the differences in the implementation of UNHS between Northern California and Rizal Province. The establishment of a tracking system, program accreditation, the rise of outsourcing services, and a decentralized regulation of newborn hearing screening was among the key differences California has from the Philippines. The existence of legislation was the only similarity between California and the Philippine UNHSP.

\section{Recommendations}

There is a need to develop a regularly updated local directory of facilities with screening, diagnostic, and intervention services that can encourage healthcare practitioners to connect their patients to more accessible institutions. Proactive coordination with uncertified centers to facilitate the certification process can also be beneficial for the surveillance, monitoring, and evaluation of the program.

The HeLe project aims to increase the newborn hearing screening rates of the Philippines through novel technology and tele-audiology. However, before the Philippines can fully adopt this tele-audiology practice, electronic billing systems should be in place that allows for the service's insurance reimbursement. Moreover, efforts toward government buy-in for the program's promotion, monitoring, and tracking system are also recommended. Blended learning techniques are also recommended by utilizing online training modules and in-person evaluation and orientation. The NHSRC would also need to certify screening personnel. Still, institutionbased programs assure the quality of services whether or not the screening is done in-house or outsourced, as some midwife clinics have started to do.

Furthermore, there is a need to establish and implement quality measures by the Department of Health and the NHSRC in consultation with professional organizations of related disciplines to achieve universal newborn hearing screening. Thus, an electronic registry and tracking system is recommended to monitor and evaluate real-time data at a national, regional, and institutional level.

\section{Acknowledgments}

This study was supported by the Philippine California Advanced Research Institute for Health, Health Innovation and Translational Medicine Project 2015-001: Increasing the Rates of Newborn Hearing Screening with Novel Technologies and Telehealth.

\section{Statement of Authorship}

All authors have substantial contributions to the conception or design of the work; or the acquisition, analysis, or interpretation of data for the work. Drafting the work or revising it critically for important intellectual content; Final approval of the version to be published; Agreement to be accountable for all aspects of the work in ensuring that questions related to the accuracy or integrity of any part of the work are appropriately investigated and resolved.

\section{Author Disclosure}

All authors declared no conflicts of interest.

\section{Funding Source}

This study was funded by the Commission of Higher Education and the Philippine California Advanced Research Institute for Health (CHED-PCARI). 


\section{REFERENCES}

1. Chiong CM. Newborn Hearing Screening in the Philippines: From Research to R.A. 9709. UPM Journal. 2011;14:30.

2. Tavartikiladze GA, Markova TG, Chibisova SS, Al'schardzhabi I, Tsygankova ER. The Russian and International Experience with the Implementation of the Programs of Universal Newborn Audiological Screening of the Newborn Infants. Vestn Otorinolaringol [Internet]. [date cited] 2016; 81(2): 7-12. Available from: https://www.ncbi. nlm.nih.gov/pubmed/27213647.

3. Scheepers LJ, Swanepoel D, le Roux T. Why Parents Refuse Newborn Hearing Screening and Default Follow-up Rescreening - A South African Perspective. Int J. Pediatr Otorhinolaryngol. 2014 January; 78: 652-8. Available from: https://www.ncbi.nlm.nih. gov/pubmed/24560238 DOI: 10.1016/j.ijporl.2014.01.026.

4. Ahmad A, Mohamad I, Mansor S, Daud MK, Sidek D. Outcome of a Newborn Hearing Screening Program in a Tertiary Hospital in Malaysia. Ann Saudi Med. 2011 January-February;31(1):24-8.

5. Huang CM, Yang IY, Ma YC, Lin GF, Yang CC, Tsai HT, et al. The Effectiveness of the Promotion of Newborn Hearing Screening in Taiwan. Int J Pediatr Otorhinolaryngol. 2014 October; 78:14-18. Available from: https://www.ncbi.nlm.nih.gov/pubmed/24300945 DOI: 10.1016/j.ijporl.2013.10.005.

6. Olusanya BO, Swanepoel DW, Chapchap MJ, Castillo S, Habib H, Mukari SZ, et al. Progress Towards Early Detection Services for Infants with Hearing Loss in Developing Countries. BMC Health Serv Res [Internet]. [date cited] 2007 January 31; 7(14). Available from: http://www.biomedcentral.com/1472-6963/7/14 DOI: 10.1186/ 1472-6963-7-14.

7. Lang-Roth R. Hearing Impairment and Language Delay in Infants: Diagnostics and Genetics. GMS Curr Top Otorhinolaryngol Head Nck Surg [Internet]. [date cited] 2014;13:1-31. Available from: https://www.ncbi.nlm.nih.gov/pubmed/25587365 DOI: 10.3205/cto000108.

8. Lammers MJ, Jansen TT, Grolman W, Lenarz T, Versnel H, van Zanten GA, et al. The Influence of Newborn Hearing Screening on the Age of Cochlear Implantation in Children. Laryngoscope. 2015; 125(4): 985-90.

9. Langer A, Brockow I, Nennstiel-Ratzel U, Menn P. The Costeffectiveness of Tracking Newborns with Bilateral Hearing Impairment in Bavaria: A Decision-analytics Model. BMC Health Serv. Res [Internet]. [date cited] 2012; 12(418) Available from: http://www. biomedcentral.com/1472-6963/12/418.

10. Santos-Cortez RLP, Chiong CM. Cost-analysis of Universal Newborn Hearing Screening in the Philippines. Acta Medica Philippina. 2013; 47(4): 52-7.

11. Reyes-Quintos MT, Isleta PD, Chiong CM, Abes GT. Newborn Hearing Screening Using the Evoked Otoacoustic Emission: The Philippine General Hospital Experience. PJOHNS. 2000; 16(4): 158-60.

12. Tan-Bumanlag RA, Romualdez JA. Initial Outcome of the Universal Newborn Hearing Screening Program at St. Lukes Medical Center. Philipp J Pediatr. 2005 January-March; 54(1): 31-7.

13. Zapanta WS, Reyes-Quintos MT. Pass and Refer Rates Using Distortion Product Otoacoustic Emissions (DPOAE) as a Screening Test among High Risk and Non-high Risk Infants: A Pilot Study. PJOHNS. 2002; 17(1-2): 38-47.

14. Abes FL, Gloria-Cruz TI, Abes GT. The Voice Test for Newborn Hearing Screening. Acta Med Philipp. 2012; 46(3): 46-51.

15. Garcia MM, Chiong CM, Abes GT, Carillo RC. Accuracy of Reflexive Behavioral ("baah") Test in the Screening for Hearing Impairment in Infants Six Months Old and Below. PJOHNS. 2012 January-June; 27(1): 6-11.

16. Santos KPA. 2018 Accomplishment Report. Newborn Hearing Screening Reference Center. January 18, 2019. Unpublished
17. Fang X, Li X, Zhang Q, Wan J, Sun M, Chang F, et al. Universal Neonatal Hearing Screening Program in Shanghai, China: An Interregional and International Comparison. Int J Pediatr Otorhinolaryngol [Internet]. 2016 August 29; 50: 77-85. Available from: http://dx.doi. org/10.1016/j.ijporl.2016.08.022.

18. Tran T, Wang, HY, Webb J, Smith MJ, Soto P, Ibieta T, Peat M, etc. Using Medicard Data to Improve New-born Hearing Screening Follow-up Reporting: Results from a Pilot Study. J Healthcare Commun [Internet]. [date cited] 2016; 1(2). Available from: http:// www.imedpub.com/ DOI: 10.4172/2472-1654.100013.

19. Bush ML, Hardin B, Rayle C, Lester C, Studts CR, Shinn JB. Rural Barriers to Early Diagnosis and Treatment of Infant Hearing Loss in Appalachia. Otol Neurotol [Internet]. [date cited] 2015 January; 36(1): 93-8. Available from: https://www.ncbi.nlm.nih.gov/pmc/articles/ PMC4268139/ DOI: 10.1097/MAO.0000000000000636.

20. Weichbold V, Welzl-Mueller K, Mussbacher E. The Impact of Information on Maternal Attitudes Towards Universal Neonatal Hearing Screening. Br J Audiol. 2001; 35: 59-66.

21. Dela Cruz ER. Resident Physicians' Knowledge, Attitudes, and Practices in Relation to Newborn Hearing Screening at Ilocos Training and Regional Medical Center. CGHH. 2016. [Internet] [date cited] Available from: http://conference.usu.edu/CGHH/Uploads/ pdfs/16371_3567EmmanuelDelaCruz.pdf.

22. Olusanya B, Luxon L, Wirz S. Maternal Views on Infant Hearing Loss in a Developing Country. Int. J. Pediatr Otorhinolaryngol. 2006 May; 70: 619-23. Available from: www.elsevier.com/locate/ijporl DOI: 10.1016/j.ijporl.2005.08.004

23. Ravi R, Yerraguntla K, Gunjawate D, Rajeshekar B, Guddattu V. Knowledge and Attitude (KA) Survey Regarding Infant Hearing Loss in Karnataka, India. Int J Pediatr Otorhinolaryngol [Internet]. [date cited] 2016 March 23; 85:1-4. Available from: http:// dx.doi.org/10.1016/j.ijporl.2016.03.012.

24. Rajagopalan R, Selvarajan HG, Rajendran A, Ninan B. Grandmothers' Perspective on Hearing Loss in Children and Newborn Hearing Screening. Indian J Otol. 2014 January; 20(1): 20-23. Available from http://www.indianjotol.org DOI: 10.4103/0971-7749.129803.

25. Nesbitt TS, Dharmar M, Katz-Bell J, Hartvigsen G, Marcin, JP. Telehealth at UC Davis - A 20-Year Experience. Telemed J E Health. 2013 May; 19(5):357-62 Available from: https://www.ncbi.nlm. nih.gov/pubmed/23343257 doi: 10.1089/tmj.2012.0284

26. Dullet NW, Geraghty EM, Kaufman T, Kissee JL, King J, Dharmar M, et al. Impact of University-Based Outpatients Telemedicine Program on Time Savings, Travel Costs, and Environmental Pollutants. Value Health. 2017 April; 20(4): 542-6 Available from: https://www. ncbi.nlm.nih.gov/pubmed/28407995 doi: 10.1016/j.jval.2017.01.014

27. Yang NH, Dharmar M, Yoo BK, Leigh JP, Kuppermann N, Romano PS, et al. Economic Evaluation of Pediatric Telemedicine Consultations to Rural Emergency Departments. Med Decis Making. 2015 August; 35(6): 773-83. Available from: https://www.ncbi.nlm. nih.gov/pubmed/25952744 doi: 10.1177/0272989X15584916.

28. Swanepoel D, HallJW.A Systematic Review of Telehealth Applications in Audiology. Telemedicine and e-Health. 2010;16(2):181-200.

29. Dharmar M, Simon A, Sadorra C, Friedland G, Sherwood J, Morrow $\mathrm{H}$, et al. Reducing Loss to Follow-Up with Tele-audiology Diagnostic Evaluations. Telemed J E Health. 2016 February: 22(2):159-64. Available from: https://www.ncbi.nlm.nih.gov/pubmed/26544032 doi: 10.1089/tmj2015.0001.

30. Rozul CD, Gregorio Jr ER, Chiong CM. Baseline Knowledge, Attitudes, and Practices of Healthcare Practitioners in Rizal Province, Philippines toward Implementing the Universal Newborn Hearing Screening Program. Acta Med Philipp. 2020 Apr 30;54(2):134-41.

31. Mackey AR, Bussé AM, Hoeve HL, Goedegebure A, Carr G, Simonsz HJ, al. Assessment of Hearing Screening Programmes Across 47 Countries or Regions II: Coverage, Referral, Follow-up and Detection Rates from Newborn Hearing Screening. Int J Audiol. 2021 Mar 9:1-10. 


\section{APPENDICES}

\section{Appendix A. Topic Guide for Rizal Participants}

\section{Knowledge}

1. How can a child have hearing loss?

2. How can hearing loss affect a child's life?

3. How does having a child with hearing loss affect a family mostly composed of hearing persons?

4. At what age can we detect deafness or hard of hearing in people?

5. How can we detect hearing loss in your specified age range?

6. We have a law mandating that newborns undergo hearing screening. What characteristics should an infant have to be eligible for hearing screening?

\section{Attitudes}

7. How necessary is the detection of hearing loss among infants?

8. What do you think is your role in the accomplishment of the newborn hearing screening program?

9. How important is it for you to accomplish your role in the newborn hearing screening program?

\section{Practices}

10. What challenges do you face in accomplishing your role in the Universal Newborn Hearing Screening Program?

11. What do you think can help in increasing the rates of newborn hearing screening in Rizal Province?

\section{Appendix B. Topic Guide for Participants in California}

1. How did the newborn hearing screening program start in California?

2. What were the challenges faced in implementing the newborn hearing screening program?

3. How is the newborn hearing screening program regulated?

4. How are newborn hearing screening centers in California sustained?

5. What are the recent developments made to increase and maintain newborn hearing screening rates here in California?

6. What are the costs of these developments?

7. What are the obstacles you encountered in developing these innovations?

8. How were you able to resolve these problems? 